\title{
A new Section of Anthurium, Sect. DeCurRentia - Revision of the Anthurium decurrens Poeppig complex in Amazonia
}

\author{
Thomas B. Croat ${ }^{1}$, Jorge Lingán ${ }^{2}$ \& Douglas Hayworth ${ }^{3}$
}

\begin{abstract}
(A new section of Anthurium, sect. Decurrentia - Revision of the Anthurium decurrens Poeppig complex in Amazonia) A revision of 6 closely related species from the Amazon basin in Colombia, Ecuador and Peru is presented, and 4 new species of Anthurium are published as new: Anthurium ceronii Croat, A. payaminoense Croat \& Lingán, A. sydneyi Croat \& Lingán and $A$. whitmorei Croat \& Lingán. Two species, A. decurrens Poeppig and $A$. longispadiceum K. Krause are redescribed because they have been, until recently, totally unknown or misrepresented by other taxa. The species are all members of a new section named Decurrentia, newly named here for the first time. All members of section Decurrentia have in common short internodes, elongated, epunctate leaf blades, and peduncles tending to be weakly or prominently ridged or winged. In addition, several of the species have early-emergent, elongated berries. All species treated here occur in the lowlands of Amazonia and have been confused with one another by botanists for a long time.
\end{abstract}

Key-words: Anthurium, new section, Decurrentia, Amazonia, new species.

\section{Resumo}

(Uma nova seção de Anthurium, sect. Decurrentia - Revisão do complexo Anthurium decurrens Poeppig na Amazônia) É apresentada uma revisão de seis espécies estreitamente relacionadas da bacia amazônica na Colômbia, Equador e Peru, sendo quatro espécies novas: Anthurium ceronii Croat, A. payaminoense Croat \& Lingán, A. sydneyi Croat \& Lingán e A. whitmorei Croat \& Lingán. Duas espécies, A. decurrens Poeppig e $A$. longispadiceum K. Krause, são novamente descritas porque foram, até recentemente, totalmente desconhecidas ou confundidas com outros taxa. As espécies são membros de uma seção nova, Decurrentia, descrita aqui pela primeira vez. Todos os membros da seção Decurrentia têm em comum internós curtos, alongados, lâminas da folha sem pontuações e pedúnculos que tendem a ser fraca ou proeminente rígidos ou alados. Além disso, várias espécies têm bagas precocemente emergentes e alongadas. Todas as espécies tratadas aqui ocorrem na planície amazônica e têm sido confundidas umas com as outras por botânicos durante muito tempo.

Palavras-chave: Anthurium, nova seção, Decurrentia, Amazônia, espécie nova.

\section{INTRODUCTION}

This paper discusses a group of species related to A. decurrens Poeppig. All are species with short internodes, elongated leaf blades, often many times longer than wide or oblanceolate, and all have peduncles that are either winged or ribbed. These species, and many others like them, do not really fit into any currently recognized section.

Schott (1860) placed A. decurrens Poepp. in his grex Oxycarpium along with $A$. oxycarpum Poepp. and $A$. consobrinum Schott, but the latter two species have both proven to be members of section Pachyneurium (Croat 1991) and Schott's description of grex Oxycarpium (as well as the fact that he chose to name the section Oxycarpium) clearly show that this sectional name should be synonymous with section Pachyneurium.

Engler (1905) adopted Schott's grex Oxycarpium, renaming it sect. Oxycarpium, and changed the section only by adding $A$. pittieri Engl. In the revision of Anthurium for Central America (Croat 1983, 1986), Croat followed the same sectional classification. The species in this presumed section have little in common overall since two species, $A$. decurrens and $A$. pittieri are not easily assigned to any other natural section of

Artigo recebido em 07/2004. Aceito para publicação em 02/2005.

${ }^{1}$ Missouri Botanical Garden. P.O. Box 299. St. Loius, MO 63166-0299, USA. e-mail: Thomas.Croat@mobot.org

${ }^{2}$ Museo Historia Natural, UNMSM, Lima, Peru.

${ }^{3}$ Washington University, St. Louis, MO, USA. 
Anthurium. The senior author has long contemplated creating a new section of Anthurium to accommodate these and other similar species and this new section is described here and revised for the Amazonian basin.

Anthurium section Decurrentia Croat, sect. nov. Type: Anthurium decurrens Poeppig (the name "decurrentia" is based on the type species).

Internodia brevia; petiolus elongatus; lamina elongata, plerumque acute ad basim, eglandulosa.

Only a few sections of Anthurium have species with elongated blades. These include Anthurium sect. Xialophyllium, which has elongated internodes, Anthurium sect. Porphyrochitonium, which has short internodes but conspicuously glandular punctuations on one or more blade surfaces, and Anthurium sect. Urospadix which has typically short internodes and blades epunctate or punctate, but with the punctuations not obviously glandular (rather more diffuse and not conspicuously rounded). It is our belief that the section Urospadix is restricted to eastern South America and the Lesser Antilles in the West Indies, and that the group is absent from the much younger Andean regions of western South America. Hopefully, molecular studies being carried out by Monica Carlsen will confirm these beliefs.

The exact number of species in section Decurrentia is at this time unknown, but there are probably many, especially in South America. Currently the only Central American species in the section is Anthurium pittieri Engl., but in addition to those species treated here there are still more undescribed species in South America, especially in the Amazon basin. This paper deals with only those species known from the Amazon basin. A future paper will describe more species in this section and list other species believed to be in section degurcens Poeppig complex

1. Leaf blade usually more than 6 times longer than wide, usually broadest at the middle, rarely above the middle.

2. Plants more or less erect; blades about twice as long as petioles; petioles only weakly sheathed at base; peduncle terete; spadix green at anthesis, long and slender, ca. 60 times longer than wide, to $25 \mathrm{~cm}$ long, $4 \mathrm{~mm}$ diam., widest at base, green; berries red at maturity. A. longispadiceum

2. Plants more or less pendent; blades 3.6-5.3 times longer than petioles; petioles conspicuously sheathed (1.2-2.3 their length); peduncles quadrangular; spadix olive (B \& K yellow 5/5) at anthesis, bluntly tapered at apex, ca. 32 times longer than wide, $6-13 \mathrm{~cm}$ long, $4 \mathrm{~mm}$ diam. at base and middle, $3 \mathrm{~mm}$ diam. at apex, turning reddish at maturity; berries white at maturity A. ceronii

1. Leaf blade $<6$ times longer than wide (sometimes $>6$ times longer than wide in $A$. longispadiceum).

3. Leaf blades with the primary lateral veins and the collective veins drying conspicuous; collective veins of leaf blade usually relatively remote from the margins; leaf blade surfaces drying blackened.

4. Stems with the cataphylls persisting intact; flowers 4 visible per principal spiral, $3-4$

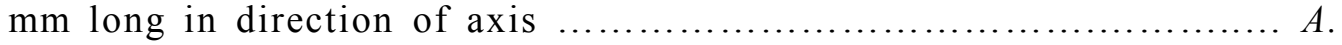
payaminoense

4. Stems with the cataphylls deciduous or persisting as fibers; flowers 6 visible per spiral, $1.5-1.7 \mathrm{~mm}$ long A. whitmorei

3. Leaf blades with the primary lateral veins and collective veins drying inconspicuous; blade surfaces drying yellowish brown (except sometimes blackened in A. longispadiceum); 
fruits early emergent and much longer than wide.

5. Spathe prominently decurrent on the peduncle; peduncle more or less as long as the petioles A. decurrens

5. Spathe not prominently decurrent, attached more or less at a single point on the peduncle; peduncle typically considerably longer than petioles.

6. Spadix more than $12.5 \mathrm{~cm}$ long, 60 times longer than wide; spathe weakly decurrent A. longispadiceum

6. Spadix less than $7.5 \mathrm{~cm}$ long, less 12 times longer than wide; spathe not at all decurrent on peduncle. A. sydneyi

Anthurium ceronii Croat, sp. nov. Type: Ecuador. Napo, $5.7 \mathrm{~km} \mathrm{~W}$ of Tena at Río Tena, $0^{\circ} 01^{\prime} \mathrm{S}, 77^{\circ} 51^{\prime} \mathrm{W}$, ca. $500 \mathrm{~m}$, T. B. Croat 58849 (holotype, MO-3154535; isotypes, AAU, NY, QCA, US). Fig. 1 a-d.

Epiphytica; internodia $0.5-1.5 \mathrm{~cm}$ longa, $0.4-1.8 \mathrm{~cm}$ diam.; cataphylla $5-8 \mathrm{~cm}$ longa, persistens intacta; petiolus $3.5-18 \mathrm{~cm}$ longus; lamina oblonga vel anguste elliptica, 30-60 cm longa, 2-4.5 cm lata; nervis primariis lateralibus $12-18$ utroque; pedunculus 16-25 cm longus, $3 \mathrm{~mm}$ diam., quadrangularis; spatha flavo-virens, $6-10 \mathrm{~cm}$ longa, $1-1.5 \mathrm{~cm}$ lata, stipitata $12-20 \mathrm{~mm}$; spadix olivaceous, $6-13 \mathrm{~cm}$ longus, $4 \mathrm{~mm}$ diam.; bacca lutea.

Epiphyte; stems pendent, ca. $20 \mathrm{~cm}$ long, to $1 \mathrm{~cm}$ diam.; internodes $0.5-1.5 \mathrm{~cm}$ long, $0.4-1.8 \mathrm{~cm}$ diam.; cataphylls $5-8 \mathrm{~cm}$, green, 1-ribbed and acuminate at apex, persisting more or less intact at upper nodes, drying brown into brittle fibers at lower nodes; Leaves with petioles $3.5-18 \mathrm{~cm}$ long, spreading-pendent, sheathed to between $1 / 2$ and 3/4 their length; geniculum upturned, thicker and drying darker than petiole; blades oblong-narrow elliptic, (6.2)16-20 cm long, 4-8 times longer than petiole, $30-60 \mathrm{~cm}$ long, $2-4.5 \mathrm{~cm}$ wide, broadest at $2 / 3$ to $3 / 4$ the length from base, moderately coriaceous, narrowly acuminate with acumen $2-4 \mathrm{~cm}$, attenuate at base, both surfaces matte, upper surface dark green, drying grayish, lower surface much paler, drying more brownish; midrib convexly raised above, much larger below; primary lateral veins $12-18$ per side, departing midrib at $35^{\circ}$ angle, raised slightly more below than above; interprimary veins only slightly less prominent than primary veins; collective veins arising from the base, with same prominence as primary lateral veins, $2-5 \mathrm{~mm}$ from margin. Inflorescence spreading, shorter than leaves; peduncle 16$25 \mathrm{~cm}$ long, $3 \mathrm{~mm}$ diam., pale green, quadrangular, the margins winged; spathe spreading, subcoriaceous, yellowish green (B $\& \mathrm{~K}$ yellow green $8 / 10$ ), linear or narrowly lanceolate, $6-10 \mathrm{~cm}$ long, $1-1.5 \mathrm{~cm}$ wide, broadest near base, inserted at a $60^{\circ}$ angle on petiole, the apex acuminate with acumen inrolled, $5 \mathrm{~mm}$ long, the base margins meeting acutely at $50^{\circ}$ angle, stipe $12-20 \mathrm{~mm}$ long in back; spadix olive-green (B\&K yellow $5 / 5$ ) at anthesis, paler (B\&K yellow 7/7.5) prior to anthesis, long ellipsoid, scarcely, bluntly tapered at apex, curved upwards away from spathe, $6-13 \mathrm{~cm}$ long, $4 \mathrm{~mm}$ diam. at base and at middle, $3 \mathrm{~mm}$ diam. at apex, turning reddish, producing white berries; flowers slightly rhombic, $2.8 \mathrm{~mm}$ long, $2.2-2.4 \mathrm{~mm}$ wide, 5 in principal spiral, 3 in alternate spiral, the sides nearly straight to slightly sigmoid; tepals matte pre-anthesis, $1.2 \mathrm{~mm}$ wide, inner margins rounded, outer margins 2-sided, pistils raised before stamens emerge, green, stigma slitlike, raised, droplets appearing several days before anthesis, $0.5 \mathrm{~mm}$ long, slightly papillate; stamens emerge in unusual manner, the alternates preceeding laterals by 2 spirals, barely emerging above tepal level, closely circling pistil but not obscuring pistil; anthers white; $0.6-0.7 \mathrm{~mm}$ long, $0.4 \mathrm{~mm}$ wide; thecae $0.2-0.3 \mathrm{~mm}$ wide; pollen white.

Anthurium ceronii ranges from southern Colombia to Ecuador and northern Peru, and ranges throughout the eastern foothills of the 
Andes Mountains in Tropical wet forest (Twf), Premontane wet forest (P-wf), and Premontane rain forest (P-rf) life zones at 450-1600 m. This epiphyte is recognized by its pendent habit, conspicuously sheathed petioles, long, narrow leaf blades, quadrangular peduncles, spreading spathe, stipitate spadix with flower tepals turning red and fruits maturing white.

The species is most easily confused with A. longispadiceum K. Krause a species with which it sometimes occurs.

Anthurium ceronii is named in honor of Ecuadorian botanist and teacher Sr. Carlos Cerón an intrepid collector of Araceae who collected much of the better material of the species.

The first collection of $A$. ceronii was made by Luis Sodiro in December, 1904 from along the Río Pastaza on the slopes of Volcán Tungurahua in what may have been an area of Lower Montane wet forest (LM-wf). Sodiro named the plant $A$. cultrifolium, but because this name was a homonym to the earlier named A. cultrifolium Schott, Sodiro's plant necessarily must have a new name.

Paratypes: COLOMBIA. PUTUMAYO: Macoa, Corregimiento de San Antonio, Vereda Alto Campucuna, Finca Mariposa, $1^{\circ} 12^{\prime} \mathrm{N}, 76^{\circ} 38^{\prime} \mathrm{W}, 1350 \mathrm{~m}$, Franco et al. 5365 (COL), Franco et al. 5404 (COL); trail between Finca La Mariposa and Alto La Sierra, $1^{\circ} 12^{\prime} \mathrm{N}, 7^{\circ} 38^{\prime} \mathrm{W}, 1500-1670 \mathrm{~m}$, Betancur et al. 5445 (COL). ECUADOR. MORONA-SANTIAGO: Cordillera del Cóndor, W slope above Valle del Río Quimi, 330'38"S, 78²4'55"W, 1600 m, 11 Dec. 2000, Freire et al. 4317 (MO, QCNE); 1300 m, 11 Dec. 2000, Pabón et al. 313 (MO, QCNE); Indanza, 305'S, 78 $25^{\circ} \mathrm{W}, 1300$ 1600 m, 23 Mar. 1974, Harling \& Andersson 12793 (GB, MO); Cordillera Cutucú, Río Itzintza, 2०40'S, 7800'W, 1500-1833 m, Camp 1316 (MO, NY); 1600-1930 m, Camp 1355 (NY); $15 \mathrm{~km} \mathrm{~N}$ of Macas, on rd. to Río Upano, $2^{\circ} 07^{\prime} \mathrm{S}, 78^{\circ} 08^{\prime} \mathrm{W}, 1250 \mathrm{~m}$, Bohlin et al. 1486 (GB). Napo: Cantón Archidona,
Hollín-Loreto, $\mathrm{km} \mathrm{25,} \mathrm{slopes} \mathrm{of} \mathrm{Volcán}$ Sumaco, 043'S, 77³6'W, 17 Dec. 1988, Hurtado 1208 (MO, QCNA); along Río Itzintza, ca. $2^{\circ} 40^{\prime} \mathrm{S}, 78^{\circ} 00^{\prime} \mathrm{W}, 1500 \mathrm{~m}$, Camp 1355 (NY); Estación Biológica Jatun Sacha, $1.5 \mathrm{~km}$ S of Río Napo, $8 \mathrm{~km}$ E of Río Puerto Misahuallí, 1 ${ }^{\circ} 04^{\prime} \mathrm{S}, 77^{\circ} 36^{\prime} \mathrm{W}, 450 \mathrm{~m}, 17$ Jan. 1987-6 Feb. 1987, Cerón 593 (MO); 24 Apr. 1987-55 May 1987, Cerón 1336 (MO); 2125 May 1987, Cerón 1398 (MO); 4 Sep. 1987, Cerón 1998 (MO); 20 Jan. 1990, Cerón et al. 8367 (QCNE); 16 Feb. 1990, Cerón et al. 8727 (MO), Miller \& Wilbert 2333 (MO); 6 Dec. 1988, Palacios 3282 (MO); 17-28 May 1989, Palacios 4309 (MO); 25 May 1991, Palacios \& Rubio 7338 (MO); 18 May 1985, Palacios et al. 401 (MO, NY, QAME, QCNE); $6 \mathrm{~km}$ from Misahuallí on Río Napo, 003'S, 77³5'W, 500 m, 18 Dec. 1986, Hammel 15988 (MO); Shushifindi, CocaLago Agrio, ca. $50 \mathrm{~km}$ NE of Coca, $0^{\circ} 15^{\prime} \mathrm{S}$, 76²0’W, 400 m, 16 Feb. 1974, Harling \& Andersson 12012; Hollín-Loreto, Río Huataraco, 2 hrs. on foot from Aldea Guagua Sumaco, $0^{\circ} 43^{\prime}$ S, $77^{\circ} 32^{\prime} \mathrm{W}$ ', Cerón \& Factos 7457 (QCNE); Tena, $1^{\circ} 00^{\prime} \mathrm{S}, 77^{\circ} 45^{\prime} \mathrm{W}, 500$ m, 20-23 Dec. 1958, Harling 3663 (S); Cantón Loreto, Parrochia San Vincente de Huaticocha, Comunidad Santa Rosa de Arapino, Bloque \#19 Triton, Pozo Santa Rosa, Freire et al. 2423 (QCNE); ca. $5.7 \mathrm{~km} \mathrm{~W}$ of Tena, Río Tena, $0^{\circ} 01^{\prime} \mathrm{S}, 77^{\circ} 51^{\prime} \mathrm{W}, 500 \mathrm{~m}, 1$ May 1984, Croat 58849 (AAU, MO, NY, QCA, US); Cantón Tena, Cabañas Chuva Urcu, 1 ${ }^{\circ} 08^{\prime} 32^{\prime \prime S}, 7^{\circ} 35^{\prime} 29^{\prime \prime} \mathrm{W}, 15-17$ Apr. 1993, Delinks et al. 289 (MO, QCNE); Estación Científica Yasuní, Pozo petrolero Daimi 2, 05' S, 76 ${ }^{\circ} 11^{\prime} \mathrm{W}, 26$ May 1988-8 June 1988, Cerón \& Hurtado 4150 (QCNE); Estación Científica Yasuní, Río Tiputini, NE of confluence of Río Tivacuno, $6 \mathrm{~km}$ E of km 44 on rd. to Maxus, $0^{\circ} 38^{\prime} \mathrm{S}, 7^{\circ} 30^{\prime} \mathrm{W}$; 200 300 m, 17 Nov. 1995, Romoleroux \& Foster 2006 (MO), 14 Apr. 1996, Romoleroux \& Foster 2193 (MO); Maxus oil rd., km 27, 0`35'S, 76²8'W, 8-15 July 1993, Dik 42 (QCNE). PASTAZA: $8 \mathrm{~km}$ from Puyo, 

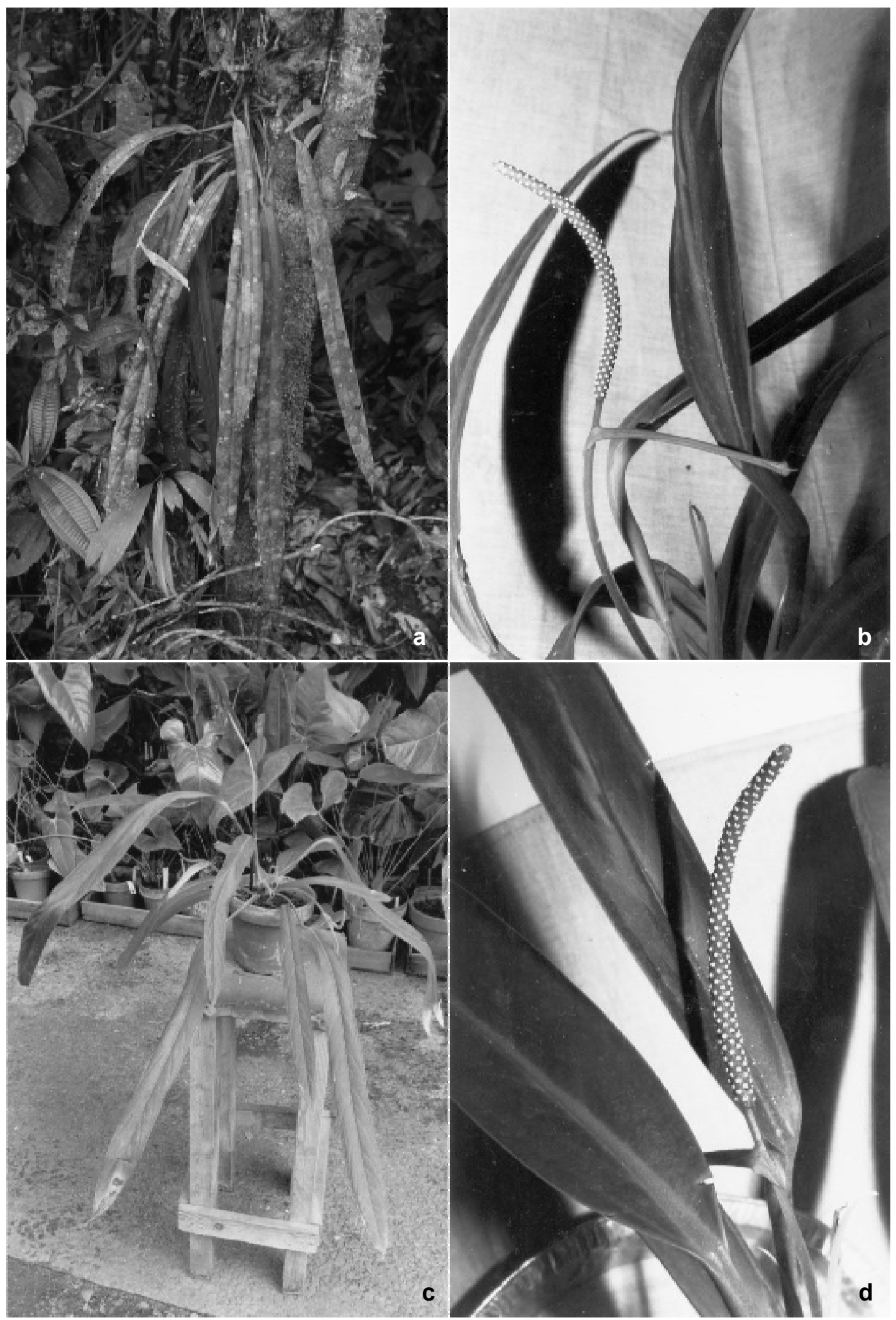

Figure 1 - Anthurium ceronii Croat: a. habit, in fruit; b. inflorescence at anthesis; c. habit in cultivation; d. inflorescence at anthesis, base of the leaf and spathe. Cultivated at Missouri Botanical Garden. (Croat 58849)

Rodriguésia 56 (88): 15-30. 2005 
$1^{\circ} 30^{\prime} \mathrm{S}, 78^{\circ} 00^{\prime} \mathrm{W}, 1000 \mathrm{~m}, 21$ July 1980 , Shemluck 305 (ECON); Puyo-Macas, $31 \mathrm{~km}$ from Puyo, $1^{\circ} 37^{\prime} \mathrm{S}, 77^{\circ} 50^{\prime} \mathrm{W}, 1100 \mathrm{~m}, 31$ Aug. 1976, Øllgaard \& Balslev 9058 (AAU); Puyo-Macas, ca. $33 \mathrm{~km} \mathrm{~S}$ of Puyo, $24.9 \mathrm{~km} \mathrm{~S}$ of Veracruz, ca. $1^{\circ} 38^{\prime} \mathrm{S}, 7^{\circ} 52^{\prime} \mathrm{W}, 900 \mathrm{~m}, 3$ May 1984, Croat 58963 (MO, QCA); Pozo petolero Villano 2 de Arco, 1 1 25'S, 1-18 Dec. 1991, Hurtado 2875 (QCNE); 4-19 Aug. 1993, Tirado et al. 50 (MO, QCNE). Sucumbios: Reserva Faunística Cuyabeno, $\mathrm{N}$ of Laguna Grande, $0^{\circ} 01^{\prime} \mathrm{N}, 76^{\circ} 11^{\prime} \mathrm{W}$, 265 m, 19 Mar. 1989, Balslev et al. 84463 (AAU); Cuyabeno Reserve, $\mathrm{N}$ of Laguna Grande, $0^{\circ} 0$ ' $\mathrm{S}, 76^{\circ} 12^{\prime} \mathrm{N}$, Nielson 76014 (AAU); 9 Nov. 1988, Paz et al. 66 (QCA); Río Aguarico, San Pablo de Secoyas, $0^{\circ} 17^{\prime}$ 'S, $76^{\circ} 26^{\prime} \mathrm{W}, 235$ m, 13 Feb. 1980, Holm-Nielsen et al. 21040 (MO); Gonzalo Pizarro, Bosque Protectora Los Cedros, Cuenca del Río Tigre, $0^{\circ} 05^{\prime} \mathrm{S}, 7^{\circ} 25^{\prime} \mathrm{W}, 17$ Mar. 1992, Tipaz et al. 771 (QCNE). TUNGURAHUA: Río Pastaza, slopes of Volcán Tungurahua, Dec. 1904, Sodiro s.n (B). PERU. LORETO: Mayna Province, Yanamono, $3^{\circ} 25^{\prime} \mathrm{S}$, $72^{\circ} 50^{\prime} \mathrm{W}, 150 \mathrm{~m}$, Aug. 1980, Croat 50124 (MO). AMAZONAS: Río Cenepa Region, Quebrada Aintami, 24 Nov. 1972, Berlin 351 (US, USM); trail E of Hampami to Shaim, 1 Aug. 1974, Berlin 1903 (USM); Prov. Condorcanqui, Cordillera del Condor, headwaters of Río Comainas, tributary of Río Cenepa, $3^{\circ} 54.1^{\prime} \mathrm{S}, 78^{\circ} 25.6^{\prime} \mathrm{W}, 1300-1500 \mathrm{~m}$, 16 July 1994, Beltrán \& Foster 850 (MO).

Anthurium decurrens Poeppig, Nov. Gen. \& Sp. 3: 83, t. 293, 1845. Type: Peru. Loreto, Maynas, Yurimaguas, Poeppig (W, destroyed). Fig. 2 a-d.

Epiphyte; internodes usually short, sometimes to as much as $1 \mathrm{~cm}$ long, $5-10 \mathrm{~mm}$ diam.; cataphylls $3.5-7.5 \mathrm{~cm}$ long, persisting intact at uppermost nodes, then cleanly deciduous; petioles (1.5) 6-22 cm long, drying 2-4 mm diam., subterete, weakly sulcate adaxially, sheathed $1-1.5 \mathrm{~cm}$, only near the base of the petiole; geniculum 1-2 $\mathrm{cm}$ long; blades linear-lanceolate to oblanceolate-elliptic, 16-36.5 cm long, 3.7$12 \mathrm{~cm}$ wide, 2.9-4.0(5.3) times longer than broad, 1.6-1.8 times longer than petioles, narrowly oblanceolate to nearly obovate, broadest usually above the middle, gradually to abruptly long-acuminate at apex; drying matte on both surfaces, dark brown to dark gray-brown above, slightly paler and grayish to yellow-brown below; midrib drying convex on both surfaces, sometimes with an acute rib on the upper surface; primary lateral veins $12-15$ per side, moderately obscure, drying scarcely more prominent than the interprimary veins, arising at a steep angle then spreading at $25^{\circ}-45^{\circ}$; all major veins scarcely raised on either surface; collective veins arising from one of the lower primary lateral veins, usually from the lowermost, sometimes from very near the base, $1-4 \mathrm{~mm}$ from the margin, not at all loop-connected. Inflorescence subpendent; peduncle $26.5 \mathrm{~cm}$ long, usually 3-4-winged, rarely terete; spathe green, erect-spreading; spadix 6-11 cm long, drying 2-5 mm diam., prominently stipitate to $2-2.3 \mathrm{~cm}$ long (stipe drying 1-1.5 $\mathrm{mm}$ diam.), green to beige, becoming purple in fruit; flowers 3 per spiral, $2.5-2.7 \mathrm{~mm}$ long, almost as wide as long; tepals broadly rounded on inner margin, 2-sided on outside margin; stamens closely aggregated around the stigma; anthers $0.4 \mathrm{~mm}$ wide, $0.3 \mathrm{~mm}$ long, yellowish, drying tan, thecae scarcely divaricate. Infructescence $25-38 \mathrm{~cm}$ long; berries prominently early-emergent and green, narrowly ovate to obovate, deep purple to red at maturity $0.6-1.5 \mathrm{~cm}$ long.

Anthurium decurrens ranges from southern Colombia to Ecuador (Napo, Pastaza), Brazil (Acre), and Peru (Amazonas, Loreto) in the Amazon basin at 225-300 (440) $\mathrm{m}$.

This species has been confused with a series of other species, especially with what has now been determined to be a new species, A. whitmorei, described in this paper. See the key to the species in this complex for key 

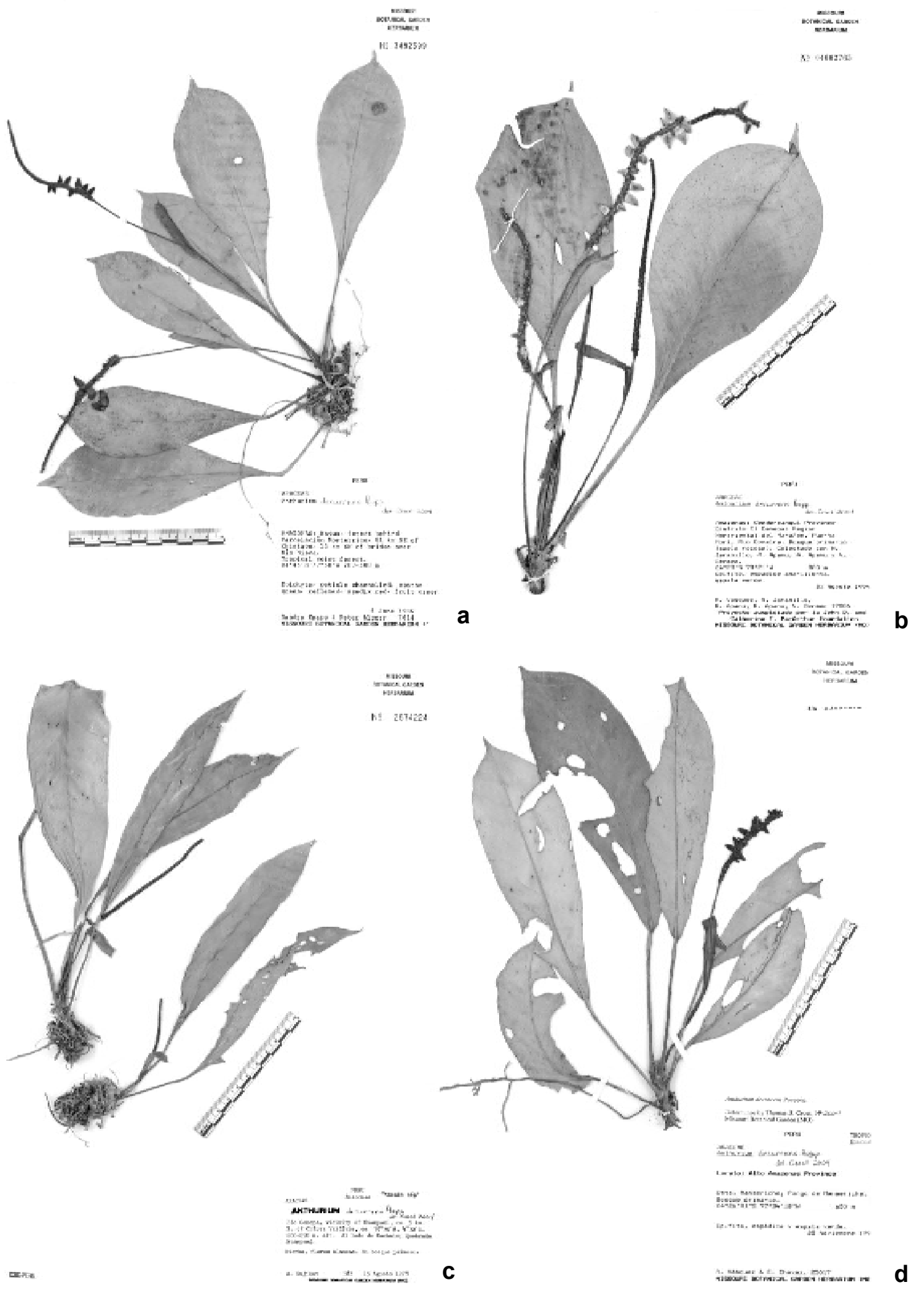

Figure 2 - Anthurium decurrens Poeppig. Herbarium specimens with inflorescences and infructescences. a. Knapp \& Alcorn 7614 (MO); b. Vásquez et al. 19006 (MO); c. Kujikat 383 (MO); d. Vásquez \& Chávez 25017 (MO). 
characters for the separation of $A$. whitmorei, and other species, from $A$. decurrens.

Additional specimens examined: PERU. AMAZONAS: Cunup, 800-850 m, Kayap 1293 (MO); Bagua, Río Santiago, 3-5 km above mouth, 250-300 m, 8-13 Oct. 1962, Wurdack 2163 (US); Imaza, PutuimShimutaz trail, $5^{\circ} 03^{\prime} 20^{\prime \prime} \mathrm{S}, 78^{\circ} 20^{\prime} 23^{\prime \prime} \mathrm{W}, 480$ m, 19 June 1994, Vásquez et al. 21261 (MO, USM); Aguaruna de Kusú-Listra, Cerro Apág, margen derecha Kusú, 600-700 m, 15 Sep. 1996, Diaz et al. 8152 (MO); Río Cenepa, 167 m, 24 Nov. 1972, Berlin 351 (MO, US, USM); Kayamas, flowing into Río Cenepa, $5 \mathrm{~km}$ N of confluence of Huampami and Río Cenepa, 213-243 m, 4 Dec. 1972, Berlin 459 (MO); trail N of Río Cenepa towards headwaters of Kayamas, 182-243 m, 18 July 1974, Berlin 1726 (MO); trail N of Río Cenepa to Tuhushiku, 213-243 m, 30 July 1974, Berlin 1881 (MO); Huampami, ca. 5 $\mathrm{km}$ E of Chávez Valdivia, ca. $4^{\circ} 30^{\prime} \mathrm{S}$, 78³0'W, 200-250 m, trail to Chigkan entsa, 8 Jan. 1978, Ancuash 1230 (MO); 5 min. downriver from Chávez Valdivia, above Tuhusik, 213-243 m, 16 Dec. 1972, Berlin 575 (MO); Huampami, Kachaig, 15 Aug. 1978, Ancuash 1491A (MO); trail to Sahim, 182-533 m, 1 Aug. 1974, Berlin 1903 (MO); $\mathrm{S}$ of Huampami, across Río Cenepa from mouth, 213-274 m, 27 Dec. 1972, Berlin 733 (MO); $5 \mathrm{~km} \mathrm{~N}$ of confluence Huampami and Río Cenepa, 256 m, 11 Oct. 1972, Berlin 231 (MO); above Huampami to $10 \mathrm{~km}$ to N, 243274 m, 22 Dec. 1972, Berlin 687 (MO); Condorcanqui, El Cenepa, Aguaruan agkiSuwa, Río Cenepa, 4³1'35"S, 78¹0’34"W, 289 m, 21 Jan. 1997, Vásquez et al. 22108 (MO, USM); El Cenepa, Kusu-kubaim, Río Comina; 425'S, 78 ${ }^{\circ} 16^{\prime} \mathrm{W}, 700$ m, 17 Aug. 1994, Vásquez et al. 18886 (MO, USM); Tutino, 4³3'05"S, 78²' '54"W, 340 m, 28 July 1997, Vásquez et al. 24493 (MO); Pumpu-entsa, 4³4'05"S, 78¹1'53"W, 26 June 1997, Vásquez 24185 (MO); Río Cenepa, vic. of Huampami, ca. $5 \mathrm{~km} \mathrm{E}$ of Chávez Valdivia, ca. 78³0’ $\mathrm{W}, 4^{\circ} 30^{\prime} \mathrm{S}, 200-250 \mathrm{~m}$,
11 Aug. 1978, Ancuash 1404 (MO); Mayaque, Río Cenepa, Saasa, 431'40'S, 78¹1'40"W, 300 m, 26 Jan. 1977, Vásquez et al. 22353 (MO, USM); Mamayaque, Cerro Sakee-gaig, 434'62"S, 78¹4'01"W, 500 m, 17 Feb. 1997 , Vásquez et al. 22613 (MO, USM). LORETO: Alto Amazonas Prov., Río Marañon, Pongo de Manseriche, Tessmann 3901 (G, NY); Cerro Campanquiz, 550-750 m, 22 Oct. 1962, Wurdack 2392 (NY); Above Pongo de Manseriche, mouth of Río Santiago, $200 \mathrm{~m}$, Mexia 6234 (US); Yurimaguas, ca. 135 m, 23 Aug.-7 Sep. 1929, Killip \& Smith 27675 (US).

Anthurium longispadiceum K. Krause, Notizbl. Bot. Gart. Berlin-Dahlem 10: 1045. 1930. Type: Colombia. Caquetá, Hetuhi, Río Orteguera, Laguna Cocha Quecachiara, Woronow \& Juzepczuk 6196 (US). Fig. 3 a-b.

Appressed epiphyte; stems short; internodes short, ca. $1 \mathrm{~cm}$ diam.; cataphylls to $10 \mathrm{~cm}$ long, $1.5 \mathrm{~cm}$ wide, broadest at base, acute at apex, drying brown and papery, soon deciduous. LEAVES spreading; petioles erect, 10-25 cm long, to $5 \mathrm{~mm}$ diam., thickest near base, terete; sheath inconspicuous; geniculum $1.5-2.0 \mathrm{~cm}$ long, infrequently drying darker than petiole, otherwise inconspicuous; blades narrowly oblongoblanceolate or rarely narrowly oblong-ovate, 1-3 times longer than petiole, moderately coriaceous, $23-36 \mathrm{~cm}$ long, $4-10 \mathrm{~cm}$ wide, 3.5-8.5 times longer than wide, usually broadest above middle, or rarely broadest below middle, drying grayish above, brownish below, short to long-acuminate at apex (acumen 1-3 cm), obtuse at base; midrib convexly raised, nearly as prominent above as below; primary lateral veins $17-25$ (occasionally fewer) pairs per side, departing midrib at $20-30^{\circ}$ angle, slightly less prominent than collective vein; collective vein arising from 2 nd or 3 rd primary lateral veins, 2-5 (10) $\mathrm{mm}$ from margin. Inflorescence erect; peduncle $16-32 \mathrm{~cm}$ long, terete; spathe green, narrowly lanceolate, 8$10 \mathrm{~cm}$ long, $1-1.5 \mathrm{~cm}$ wide, widest at base, 


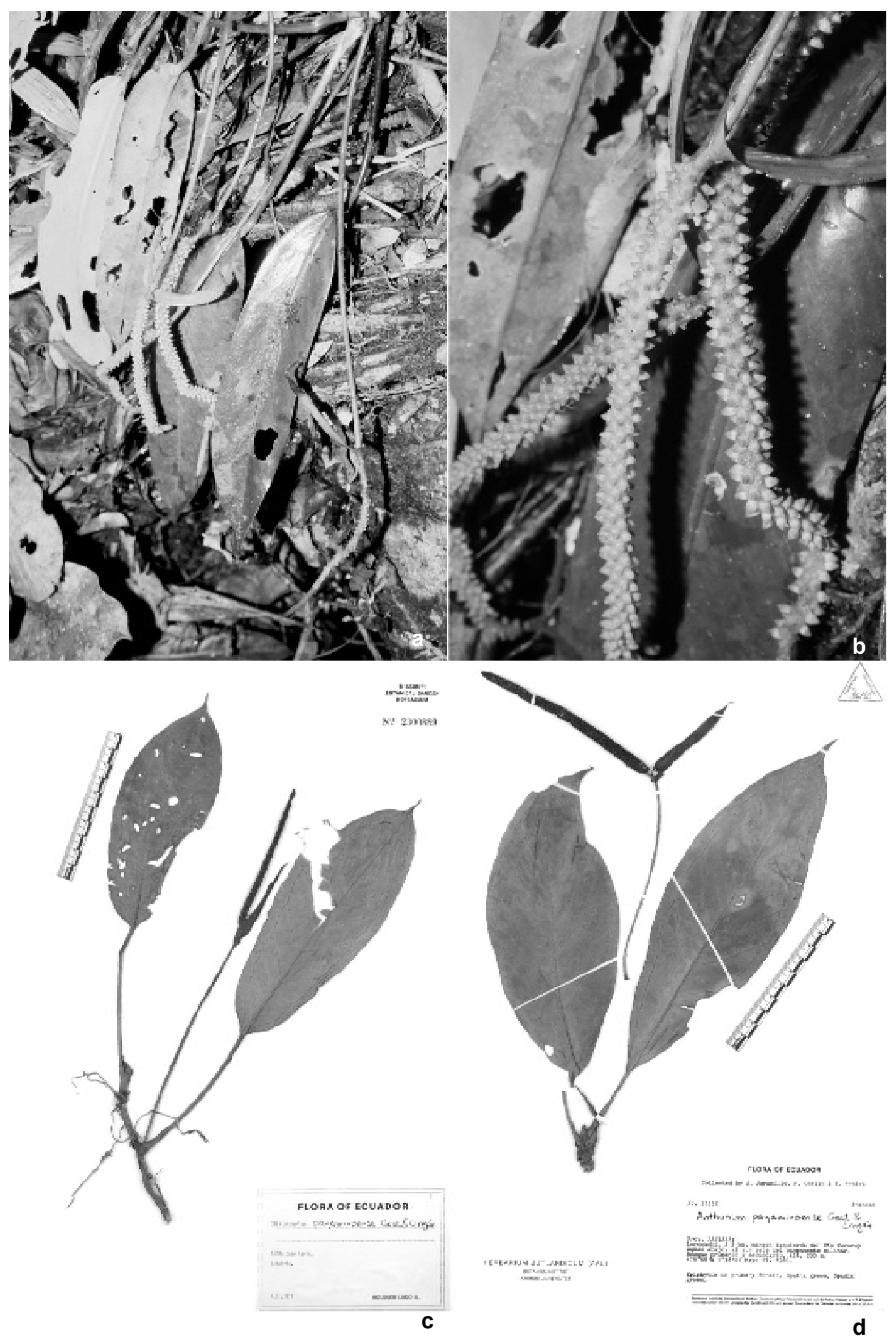

Figure 3 - a-b. Anthurium longispadiceum K. Krause: a. habit, in fruit; b. infructescences, note the berries promptly protruding. (Croat 72550); c-d. Anthurium payaminoense Croat \& Lingán: c. type specimen (Lugo 3164); d. herbarium specimen (Jaramillo 3133). 
reflexed, inserted at $70-80^{\circ}$ angle, margins meeting narrowly acute, stipe $5 \mathrm{~mm}$ long (to $1 \mathrm{~cm}$ long in fruit); spadix green at anthesis, 12.5-25 cm long, $4 \mathrm{~mm}$ diam., widest at base; flowers square to narrowly rhombic, 4 in principal spiral, $2.5 \mathrm{~mm}$ long, 1.5-2.0 mm wide, sides straight; tepals $1.5 \mathrm{~mm}$ long, $0.5-0.8 \mathrm{~mm}$ wide, inner margins rounded, outer margins 2 sided. Infructescence to $40 \mathrm{~cm}$ long, $2.5 \mathrm{~cm}$ diam., \pm pendent; berries \pm tapered and pointed as they emerge, maturing red.

Anthurium longispadiceum is known from southern Colombia, eastern Ecuador and northern Peru, occurring in lowland Tropical moist forest (T-mf) and in a small region of Tropical wet forest (T-wf) centered at $1^{\circ} 04^{\prime} \mathrm{S}$, $77^{\circ} 30^{\prime} \mathrm{W}$, at $200-575 \mathrm{~m}$. The species is distinguished by its erect habit with slender blades about twice as long as petioles, the weakly sheathed petioles, the very long greenish spadix and red berries.

Is a member of sect. Decurrentia and is distinguished from $A$. decurrens by its much narrower blades which are less conspicuously broadened toward the apex and by its proportionately longer spadices which are proportionately less stipitate. Anthurium longispadiceum is most closely related to $A$. ceronii, and is distinguished from that species by its less pendent habit, lower blade-topetiole length ratio, weakly sheathed (vs. conspicuously sheathed) petioles, terete (vs. quadrangular) peduncles, and its long, slender, green spadices with red berries (vs. red spadices with white berries for $A$. ceronii).

The species was first collected in the type locality in Colombia by G. Woronow and S. Juzepczuk, but has not been collected in that country since. It was first collected in Ecuador by H. Lugo in 1972 while collecting for the Rijks herbarium in Stockholm. While it has apparently been collected only once in Colombia and Peru, it has been collected many times in Ecuador.

Specimens examined: ECUADOR. NAPO: Orellana Cantón, Maxus Pipeline Rd., km 19, 0³3'S, 76³1'W, 230 m, 1-17 Apr. 1993,
Hurtado 3068 (MO); Aguarico Cantón, Río Aguarico, Reserva Faunística Cuyabeno Reserve, Zancudo, 0²9'S, 230 m, 25 Sep. 1991, Palacios et al. 7585 (MO); Reserva del Batallón de Selva, 005'S, 7552’W, 200 m, Aug. 1980, Andrade 33087 (AAU, QCA, QNA); $20 \mathrm{~km} \mathrm{~W}$ of Loreto, 045' $\mathrm{S}, 7^{\circ} 28^{\prime} \mathrm{W}$, 575 m, 10 Jan. 1989, Hurtado et al. 1391 (MO); Estación Biológica Jatun Sacha, 8 km E of Misahuallí, along Río Napo, $1^{\circ} 04^{\prime} \mathrm{S}$, 77³6’W, 450 m, 17 Jan. 1987-6 Feb. 1987, Cerón 865 (MO); 21-25 May 1987, Cerón 1475 (MO), 8 Nov. 1987, Cerón 2645 (MO); 6 Aug. 1989, Cerón 7236(MO); 23 May 1992, Gudiño \& Zuleta 1668 (QCNE); $1^{\circ} 08^{\prime} \mathrm{S}$, 77³0’W, 450 m, Palacios 2645 (MO), 2427 Aug. 1988, Palacios 2764 (MO), 24-27 Aug. 1988, Palacios 4326 (MO); canopy walkway, 13 Jan. 1999, Delinks \& Suárez 176 (MO, QCNE). Orellana Cantón, Río Yasuní, Nacional Yasuní, km 3 of NPF-Puerto Maxus branch, $0^{\circ} 41^{\prime} \mathrm{S}, 76^{\circ} 25^{\prime} \mathrm{W}, 250 \mathrm{~m}, 15$ June 1994, Pitman 283 (MO, QCNE); Maxus pipeline rd., $\mathrm{km} 27,0^{\circ} 35^{\prime} \mathrm{S}, 7^{\circ} 30^{\prime} \mathrm{W}, 250 \mathrm{~m}$, 4-27 July 1993, Aulestia 17 (MO), 4-27 July 1993, Aulestia 55 (MO); km 45, 045'S, 76²8'W, 230 m, 8-15 Aug. 1993, Dik 73 (MO, QCNE); pozo petrolero Diami 2, $0^{\circ} 55^{\prime} \mathrm{S}, 76^{\circ} 11^{\prime} \mathrm{W}, 200 \mathrm{~m}, 26$ May 1988-8 June 1988, Cerón \& Hurtado 4179 (MO, US); Parque Nacional Yasuní, Estación Cientifica Yasuní, vic. Tipuanti, 0³8'S, 76³0'W, 200 m, 10 Mar. 1996, Kjaer-Pedersen 2051 (MO); Yasuní Forest Reserve, vic. Pontificia Universidad Católica Field Station, 040.853'S, 76²3.697'W, 225 m, 19 June 1995, Acevedo-Rodrígues \& Cedeño 7411 (MO); ca. $80 \mathrm{~km}$ upriver from Nuevo Rocafuerte, $0^{\circ} 30^{\prime} \mathrm{S}, 76^{\circ} 00^{\prime} \mathrm{W}, 225 \mathrm{~m}, 16 \mathrm{Sep}$. 1977, Foster 3691 (F), 17 Sep. 1977, Foster 3718 (F, MO); 2-5 km WSW of San Pablo de los Secoyas, 0¹5'S, 76²1'W, 300 m, 29 Aug. 1981, Brandbyge et al. 36242 (AAU, QCA, QNA); 6 Aug. 1980, Brandbyge et al. 32519 (AAU, QCA, QNA); Río Wai si ayá, 1 $\mathrm{km}$ upstream from Río Aguarico, $0^{\circ} 15^{\prime} \mathrm{S}$, $76^{\circ} 21^{\prime} \mathrm{W}, 300$ m, 6 Aug. 1981, Brandbyge et 
al. 33206 (AAU); Aguarico Cantón, Reserva Etnica Huaorani, Maxus oil rd., km 61, S of Río Tivacuno, $0^{\circ} 48^{\prime} \mathrm{S}, 76^{\circ} 23^{\prime} \mathrm{W}, 250 \mathrm{~m}$, Aulestia et al. 984 (MO), Pitman 249 (MO, QCNE); Santa Cecilia, Río Aguarico, 004'N, 7658’W, 220 m, Sparre 13052 (S); 4-5 km $\mathrm{N}$ of Santa Rosa, on Río Bueno, $0^{\circ} 55^{\prime} \mathrm{S}$, $77^{\circ} 28^{\prime} \mathrm{W}$, ca. 400 m, Lugo 2153 (MO); Tena region, Asplund 9217 (S); Orellana Cantón, Yuca-Taracoa de Esperanza, $22.5 \mathrm{~km}$ E of jet. with main Coca-Río Tigüino Rd., 0³4'N, $6^{\circ} 42^{\prime} \mathrm{W}, 350 \mathrm{~m}$, Croat 72550 (MO); CocaLoreto-Hollín, Sitio Huaticocha, $0^{\circ} 45^{\prime} \mathrm{S}$, 77²9’W, 500 m, Palacios et al. 3545 (MO), Palacios et al. 3566 (MO). PASTAZA: Río Curaray, near Laguna Patoamo, $1^{\circ} 30^{\prime} \mathrm{S}$, $76^{\circ} 30^{\prime} \mathrm{W}, 230 \mathrm{~m}$, Neill \& Palacios 6817 (MO); Ceilán, path Ceilán-Río Cononaco, $\mathrm{S}$ of Río Curaray, $1^{\circ} 36^{\prime} \mathrm{S}, 75^{\circ} 40^{\prime} \mathrm{W}, 200 \mathrm{~m}$, Brandbyge \& Asanza 31778 (AAU), 31759 (AAU), 31779 (AAU); E of Río Añango, 400 $500 \mathrm{~m}, 1^{\circ} 30^{\prime} \mathrm{S}, 77^{\circ} 15^{\prime} \mathrm{W}, 9$ Aug. 1980, Jaramillo \& Coello 3312 (QCA); Puyo; Pambayucua, Rio Lligino, $77^{\circ} 22^{\prime} \mathrm{W}, 0^{\circ} 29^{\prime} \mathrm{S}$, 420 m, Palacios 10164 (MO, QCNE); Centro-Oriente, Población Waorani (Aucas), 400500 m, Jaramillo \& Coello 3313 (QCA). SUCUMBIOS: Lago Agrio Cantón, Lago Agrio-Baeza, km 46, $9.3 \mathrm{~km}$ E of Lumbaquí,

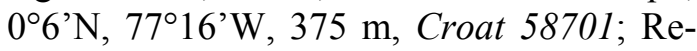
serva Faunística Cuyabeno, between Pacuyacu \& Juanillas, 0`33'S, 75³0’W, 230 m, Palacios et al. 7893 (MO). PERU. AMAZONAS: $5 \mathrm{~km}$ E of Chávez Valdivia, Río Cenepa, 200-250 m, 430' S, 78 $30^{\circ} \mathrm{W}$, Ancuash 1230 (MO).

Anthurium payaminoense Croat \& Lingán, sp. nov. Type: Ecuador. Lago Agrio, 4 Nov. 1973, Lugo 3164 (holotype, MO; isotype, GB). Fig. 3 c-d.

Epiphytica; internodia $0.5-3.2 \mathrm{~cm}$ longa, 0.4-0.7 cm diam.; petiolus $3.9-13.6 \mathrm{~cm}$ longus; lamina 16.5-30.8 cm longa, 5.5-11 cm lata, oblongo-elliptica vel oblanceolata; nervis primariis lateralibus (5)7-9 utroque; pedunculus 10.9-32.1 cm longus, 3-4-latus; spatha viridis, (3.4) 6.5-13 cm longa, 0.5-1.6 cm lata, anguste oblonga; spadix (0.2) 3.7$14.4 \mathrm{~cm}$ longus, $0.2-0.6 \mathrm{~mm}$ diam. viridis, stipitatus (0.4) 0.6-1.4 (3.2) cm.; bacca $3 \mathrm{~mm}$ longa.

Description based on dried material. Epiphyte; roots $1 \mathrm{~mm}$ diam., whitish to pale brown; stem terete; internodes $0.5-3.2 \mathrm{~cm}$ long, 0.4-0.7 cm diam; cataphylls 0.7-4 (9) $\mathrm{cm}$ long, lanceolate, membranaceous, persisting reddish brown to dark brown. Leaves erect to spreading; petioles 3.9-13.6 cm long, 0.2-0.3 cm diam., U-shaped, shallowly and acutely sulcate above, 0-2ribbed below, green; sheath 1.4-3.2 $(5.1) \mathrm{cm}$ long; geniculum 1.2-2.7 cm long; blades papiraceous, $16.5-30.8 \mathrm{~cm}$ long, $5.5-11 \mathrm{~cm}$ wide, oblong-elliptic to oblanceolate, abruptly acuminate at apex, drying dark olive green above, blackish below; midrib conspicuously raised in both surfaces, more conspicuously above; primary lateral veins (5)7-9 per side, conspicuously raised below, straight, departing at $30-45^{\circ}\left(50^{\circ}\right)$ from the midrib; collective veins arising from the base, (0.2) $0.7-1.5 \mathrm{~cm}$ from the margin. Inflorescence erect to spreading; peduncle $10.9-32.1 \mathrm{~cm}$ long, 0.1-0.3 cm diam., markedly 3-4-sided, green, 2.3-2.8 times longer than the petiole; spathe papiraceous, green, persistent, erect to spreading, (3.4) $6.5-13 \mathrm{~cm}$ long, 0.5-1.6 cm wide, narrowly oblong, conspicuously acuminate at apex, the margins joining at ca. $90^{\circ}$ angle; spadix slightly tapered at apex, (0.2) 3.7-14.4 cm long, 0.2-0.4 cm wide near to apex, $0.2-0.6 \mathrm{~mm}$ wide near to base, green; stipe greenish, (0.4) 0.6-1.4 (3.2) cm long; flowers square, margins slightly sigmoid, $2 \times$ $2 \mathrm{~mm}$; 3-4 flowers visible in the principal spiral, 5-6 flowers visible in the alternate spiral; tepals with the inner margins straight to convex; pistils with stigmas elliptic, not protruding; stamens ca. $1 \mathrm{~mm}$ long, flattened; thecae not divaricated. Infructescence erect to spreading, green; berries ca. $3 \mathrm{~mm}$ long. Anthurium payaminoense is known from 
the Provinces of Napo and Pastaza (Ecuador), ranging from $200-500 \mathrm{~m}$ in elevation and seems to be endemic. There is a voucher from Río Moa, Acre in Brazil (Jangoux et al. 85101), which looks very similar to $A$. payaminoense, but the cataphylls are totally deciduous.

Anthurium payaminoense is a member of sect. Decurrentia and is distinguished by its oblong-elliptic to oblanceolate leaves drying blackish with the collective veins not close to the margins, cataphylls persisting brown and a stipitate green spadix. It can be confused with $A$. whitmorei, which has fibrous cataphylls, longer peduncles and its blades drying greenish.

This species is named after the village of Payamino, where it is a common species.

Paratypes: ECUADOR. PASTAZA: Lorocachi, $3 \mathrm{~km}$ left bank of Río Curacay, SE of military camp, $1^{\circ} 38^{\prime} \mathrm{S}, 7^{\circ} 58^{\prime} \mathrm{W}, 200 \mathrm{~m}$, 27 May 1980, Jaramillo et al. 31332 (AAU); Pastaza Cantón, Parroquia Curacay, Pozo Petrolero Villano 2 de ARCO, between Iquino and Villano, $1^{\circ} 29^{\prime} \mathrm{S}, 77^{\circ} 27^{\prime} \mathrm{W}, 350 \mathrm{~m}, 10$ Aug. 1993, Tirado et al. 130 (CAS, CM, ENCB, MO, QCNE). NAPO: Project of Payamino, Ministerio de Agricultura y Ganadería, near roadside, $0^{\circ} 26^{\prime} \mathrm{S}, 77^{\circ} 01^{\prime} \mathrm{W}$, 200 m, 25 Feb. 1980, Brandbyge \& Asanza 30027 (AAU); Reserva El Chuncho rd. Payamino-Loreto, $0^{\circ} 10^{\prime} \mathrm{S}, 7^{\circ} 03^{\prime} \mathrm{W}, 13 \mathrm{Apr}$. 1988, Arguello 828 (QCA); Reserva Biológica Jatun Sacha, $8 \mathrm{~km}$ from Puerto Misahuallí, right bank of Río Napo, $1^{\circ} 04^{\prime} \mathrm{S}, 7^{\circ} 36^{\prime} \mathrm{W}, 450$ m, 4 Sep. 1987, Cerón et al. 2001 (MO).

Anthurium sydneyi Croat \& Lingán, sp. nov. Type: Peru. Loreto, campamento petrolero, Río Pastaza, N de Iquitos, $2^{\circ} 55^{\prime} \mathrm{S}, 76^{\circ} 25^{\prime} \mathrm{W}$, 210 m, 21 Nov. 1980, R. Vásquez \& Jaramillo 852 (holotype, MO-3032816; isotype USM). Fig. 4 a-b.

Epiphytica; internodia $0.2-0.6 \mathrm{~cm}$ longa, 0.4-0.7 cm diam.; petiolus 2.3-9.2(12.6) cm longus; lamina 8.1-23 cm longa, 2.1-6 cm lata; nervis primariis lateralibus (5)7-10 utroque; pedunculus inferme costatus, 4-20 cm longus, $0.1 \mathrm{~cm}$ diam.; spatha viridis, 1.9$6.6 \mathrm{~cm}$ longa, 0.6-1.3 cm lata, ovatolanceolata; spadix, 3.6-7.2 cm longus, 0.2$0.3 \mathrm{~cm}$ diam.

Description based on dried material; epiphytic; roots $1-2 \mathrm{~mm}$ diam., grayish to pale brown; stem terete; internodes $0.2-0.6 \mathrm{~cm}$ long, $0.4-0.7 \mathrm{~cm}$ diam.; cataphylls $2-6.2 \mathrm{~cm}$ long, 1-ribbed, oblong-lanceolate, membranaceous, brown to greenish brown, the uppermost persisting, then deciduous. Leaves erect to spreading; petioles 2.3-9.2 (12.6) cm long, 0.1-0.2 diam., U-shaped, shallowly and acutely sulcate, green; sheath 0.2-1.7 cm long; geniculum 0.2-0.5 cm long, inconspicuous; blades subcoriaceous, 8.1-23 cm long, 2.1-6 cm wide, wider at the upper third, oblanceolate to elliptic, attenuate at base, abruptly acuminate to acute at apex, semiglossy above, glossy below; midrib inconspicuously raised on both surfaces; primary lateral veins (5)7-10 per side, almost inconspicuous, straight, departing at $40^{\circ}-50^{\circ}$ angle from the midrib; interprimary veins slightly raised in both surfaces; collective veins arising from one of the $1^{\text {st }}$ or $2^{\text {nd }}$ primary lateral veins, $0.2-0.8 \mathrm{~cm}$ from the margins. Inflorescence erect; peduncle weakly ribbed, 4-20 cm long, $0.1 \mathrm{~cm}$ diam., greenish, 1.7-2.2 times longer than the petiole; spathe green, membranaceous, 1.9$6.6 \mathrm{~cm}$ long, 0.6-1.3 cm wide, ovatelanceolate, abruptly acuminate at apex, acute at base; the margins forming ca. $50^{\circ}$ angle on peduncle; spadix cylindric to slightly longtapered, green, $3.6-7.2 \mathrm{~cm}$ long, $0.2-0.3 \mathrm{~cm}$ diam.; flowers rhombic, margins straight, $4 \times$ $2 \mathrm{~mm}$; 3-4 flowers visible in both spirals; pistils $2 \mathrm{~mm}$ long; stigmas oblong; stamens ca. $2 \mathrm{~mm}$ long, filaments flattened; thecae slightly divaricate. Infructescence erect to spreading; spadix (3) 7.4-10.6 cm long, 0.7$2.3 \mathrm{~cm}$ wide; berries green to red, early emergent, ovate with the apex conical, stigmas protruding.

Anthurium sydneyi is known only from 

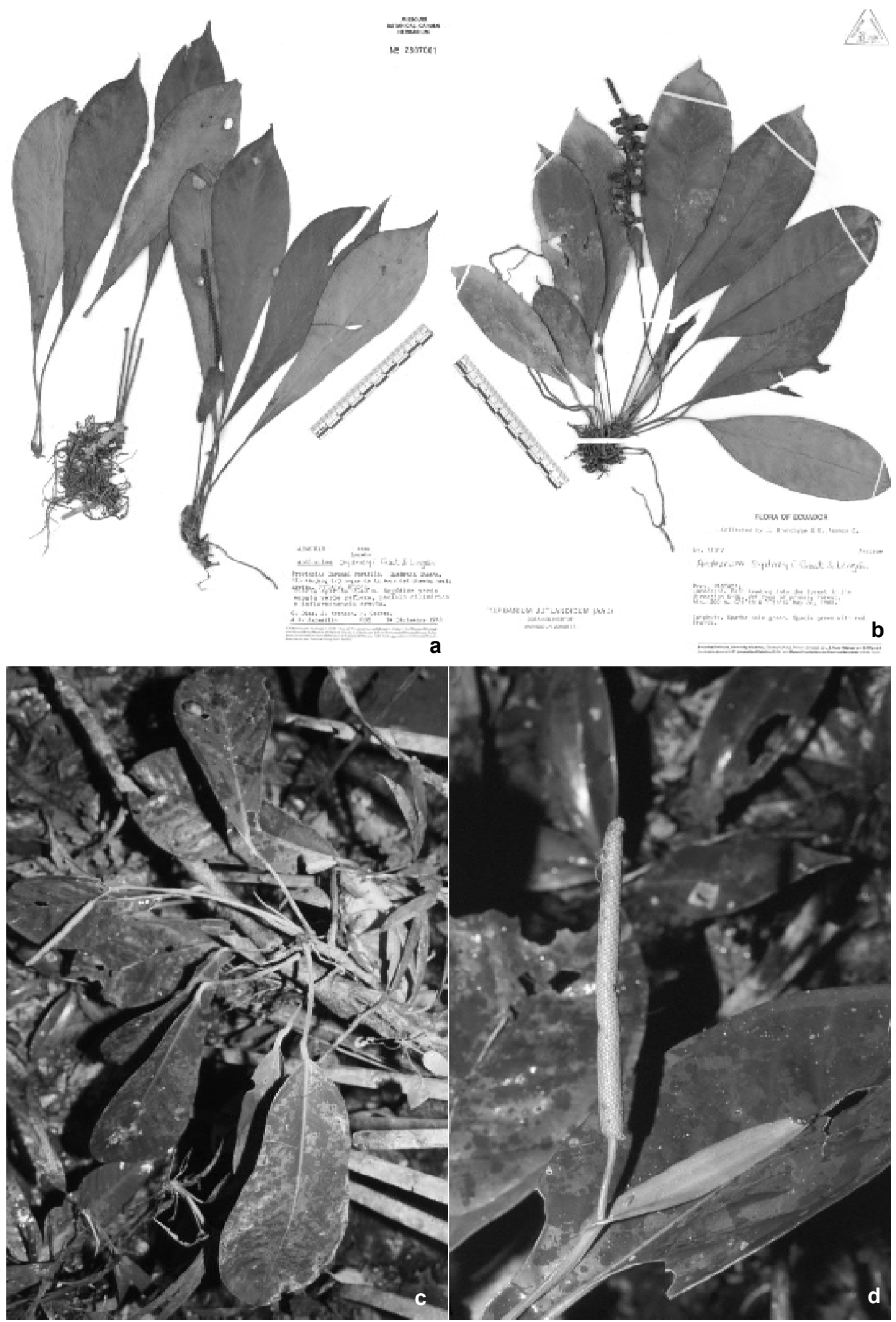

Figure 4 - a-b. Anthurium sydneyi Croat \& Lingán. Herbarium specimens at MO. a. Díaz 835; b. Brandbyge 31012; c-d. Anthurium whitmorei Croat \& Lingán. c. habit; d. inflorescence, post-anthesis. (Croat 72524)

Rodriguésia 56 (88): 15-30. 2005 
Ecuador (Pastaza Province) and Peru (Department of Loreto) in areas of Tropical moist forest (T-mf) at 180-200 m.

The species is a member of sect. Decurrentia and is distinguished by its epiphytic habit, oblanceolate leaf blades conspicuously acuminate at apex, peduncle weakly ribbed, spathe and spadix green and principally by its berries promptly emerging even before reaching maturity. This species could be confused with $A$. decurrens, but $A$. sydneyi has a spathe that is not conspicuously decurrent on the petiole.

The species is named in honor of Dr. Sidney McDaniel who collected the species in Loreto, Peru. Sidney McDaniel is one of the foremost experts on the plants of the Department of Loreto in Peru where he spent years collecting in preparation for the Flora of Loreto.

Paratypes: ECUADOR. PASTAZA: Lorocachi, on the path to Lagartococha, $1^{\circ} 38^{\prime} \mathrm{S}, 75^{\circ} 58^{\prime} \mathrm{W}, 200 \mathrm{~m}, 25$ May 1980 , Jaramillo et al. 30969 (AUU); Pica to Lagartococha, $1 \mathrm{hr}$. following the right bank of Río Curaray, S of military camp, $1^{\circ} 39^{\prime} \mathrm{S}$, $75^{\circ} 59^{\prime} \mathrm{W}, 1$ June 1980, Jaramillo et al. 31749 (AAU); path leading into the forest in the direction SSW, $1^{\circ} 38^{\prime} \mathrm{S}, 75^{\circ} 58^{\prime} \mathrm{W}, 200 \mathrm{~m}, 26$ May 1980, Brandbyge \& Asanza 31012 (AAU); Ceilán, path going from Ceilán to Río Cononaco on the S side of Río Curaray, $1^{\circ} 36^{\prime} \mathrm{S}, 75^{\circ} 40^{\prime} \mathrm{W}, 200 \mathrm{~m}, 7$ June 1980 , Brandbyge \& Asanza 31793 (AAU); 6 June 1980, Brandbyge \& Asanza 31760 (AAU). PERU. LORETO: Alto Amazonas, Andoas, $2^{\circ} 55^{\prime} \mathrm{S}, 76^{\circ} 25^{\prime} \mathrm{W}, 180 \mathrm{~m}, 3$ Nov. 1983 , Vásquez \& Jaramillo 4564 (MO); Coronel Portillo, Quebrada Shesha, Río Abujao, 1.3 hrs. from the mouth of Shesha, $8^{\circ} 20^{\prime} \mathrm{S}$, 734'' W, 14 Dec. 1978, Díaz et al. 835 (MO); Valseca-Rudolpho, Río Corrientes, between Platanoyacu \& mouth of Río Macusari, 17 Sep. 1968, McDaniel \& Marcos 11054 (MO); Loreto, Pampa Hermosa and vicinity, Río Corrientes, $1 \mathrm{~km} \mathrm{~S}$ of jct. with Río Macusari, $30^{\circ} 15^{\prime} \mathrm{S}, 75^{\circ} 50^{\prime} \mathrm{W}, 160 \mathrm{~m}$, 7 June 1986 , Lewis et al. 10843 (MO); Río Macusari, McDaniel \& Marcos 11026 (IBE). HUÁNUCO: Pachitea, Codo de Pozuzo, trail W of settlement to lower mountains slopes, $75^{\circ} 28^{\prime} \mathrm{W}, 9^{\circ} 40^{\prime} \mathrm{S}, 500-1000 \mathrm{~m}, 17$ Oct. 1982 , Foster 9244 (MO). SAN MARTÍN: Mariscal Cáceres, Madre Mía, 760-880 m, 16 Mar. 1977, Boeke 1310 (MO). 
Anthurium whitmorei Croat \& Lingán, sp. nov. Type: Ecuador. Orellana, Tiputini Biodiversity Station, $0^{\circ} 38^{\prime} \mathrm{S}, 76^{\circ} 09^{\prime} \mathrm{W}, 200$ m, 21 Feb. 2002, N. Köster et al. 1018 (holotype, MO; isotypes, BONN, K, QCA, QCNE, QUSF). Fig. 4 c-d.

Epiphytica; internodia $0.3-2.1 \mathrm{~cm}$ lon$\mathrm{ga}, 0.2-0.8 \mathrm{~cm}$ diam.; petiolus $2.7-12.7 \mathrm{~cm}$ longus, $0.2-0.5$ diam., D-formatus, vaginatus 1.9-4.2 cm; lamina $14.2-34.9 \mathrm{~cm}$ longa, $3.1-$ $10(12.6) \mathrm{cm}$ lata, oblanceolata vel oblongooblanceolata, raro obovate; nervis primariis lateralibus (7) 10-13 utroque; pedunculus 4costatus, $13.2-31 \mathrm{~cm}$ longus, $0.2-0.5 \mathrm{~cm}$ diam.; spatha viridis $4.2-13.3 \mathrm{~cm}$ longa, 0.6$1.2 \mathrm{~cm}$ lata, oblongo-lanceolata vel oblongoelliptica; spadix flavo-virens vel olivaceous, $4.5-14.8 \mathrm{~cm}$ longus, $0.2-0.6 \mathrm{~cm}$ diam., stipitatus $0.9-4.1 \mathrm{~cm}$; bacca viridis, obovata vel sphaericus, $3 \mathrm{~mm}$ longa, $2 \mathrm{~mm}$ lata.

Description based on dried material; epiphytic; roots 1-2 mm diam., grayish white to brownish; internodes $0.3-2.1 \mathrm{~cm}$ long, grayish to brown, $0.2-0.8 \mathrm{~cm}$ diam.; cataphylls $2-6.2 \mathrm{~cm}$ long, un-ribbed, lanceolate, subcoriaceous, grayish to dark brown, weathering in to brown fibers, then deciduous. Leaves erect to spreading; petioles $2.7-12.7 \mathrm{~cm}$ long, $0.2-0.5 \mathrm{~mm}$ diam., Dshaped with erect margins above, frequently bluntly ribbed below, greenish; sheath 1.9$4.2 \mathrm{~cm}$ long; geniculum $0.7-2.3 \mathrm{~cm}$ long; blades subcoriaceous, $14.2-34.9 \mathrm{~cm}$ long, $3.1-10(12.6) \mathrm{cm}$ wide, oblanceolate to oblong-oblanceolate, rarely obovate, abruptly acuminate at apex, acute to obtuse at base, semiglossy above, glossy below; margins slightly concave below the middle; midrib convex above, prominently raised below; primary lateral veins (7) 10-13 per side, slightly curved, ascending, departing midrib at $50-60^{\circ}$; collective veins arising from the base, $0.4-1.7 \mathrm{~cm}$ from the margins. Inflorescence erect to spreading; peduncle markedly 4-ribbed, $13.2-31 \mathrm{~cm}$ long, $0.2-0.5$ cm diam., green, 2.4-4.8 times longer than the petiole; spathe membranaceous to weakly subcoriaceous, green, persistent, erect, $4.2-$ $13.3 \mathrm{~cm}$ long, $0.6-1.2 \mathrm{~cm}$ wide, oblonglanceolate to oblong-elliptic, acuminate at apex, acute at base, the margins joining at 25$40^{\circ}$; spadix yellowish green to olive-green, $4.5-14.8 \mathrm{~cm}$ long, $0.2-0.6 \mathrm{~cm}$ diam.; stipe greenish, $0.9-4.1 \mathrm{~cm}$ long in front, $0.3-3.1$ $\mathrm{cm}$ long in back; flowers rhombic $1.5-1.7 \mathrm{~mm}$ long, 1.7-2 mm wide; 3-4 flowers visible on the principal spiral, 3-7 flowers visible on the alternate spiral; tepals grayish to brownish, rugulose on drying, outer margins 2-3 sided, inner margins convex; stigmas elliptic; stamens contiguous at anthesis, closely clustered over stigma; anthers $0.35 \mathrm{~mm}$ long, $0.5 \mathrm{~mm}$ wide; thecae ovoid, markedly divaricated, drying yellow-brown. Infructescence spreading; berries green, obovate to spherical, $3 \mathrm{~mm}$ long, $2 \mathrm{~mm}$ wide. Anthurium whitmorei is known from Ecuador (Napo and Pastaza Provinces) and Peru (Loreto Department), ranging from 140 to $700 \mathrm{~m}$ elevation in Tropical moist forest (T-mf).

Anthurium whitmorei is a member of sect. Decurrentia and is characterized by its cataphylls weathering into fibers, oblanceolate leaves with margins slightly concave as well as by the conspicuously 4-sided, markedly 4ribbed peduncle and a prominently decurrent spathe and the green, stipitate spadix.

There are collections that share geographical distribution and morphological features (shape of leaves, peduncle 4-ribbed, spadix stipitate), but they are greenish on drying with lanceolate spathe and slender spadix; probably they are the same species or a hybrid.

Anthurium whitmorei is named in honor of Timothy Charles Whitmore who first collected this species in Tiputini Biodiversity Station, Orellana (Ecuador).

Paratypes: ECUADOR. NAPO: Limoncocha, near NW corner of lake, Oct. 1969, Mowbray 69018 (MO); Lower Río Aguarico (above puesto military Puerto Loja), 7 Mar. 1968, Harling et al. 7401 (GB); Río 
Aguarico, San Pablo de los Secoyas, $0^{\circ} 17^{\prime} \mathrm{S}$, $76^{\circ} 26^{\prime} \mathrm{W}, 13$ Feb. 1980, $235 \mathrm{~m}$, Brandbyge et al. 21040 (AAU); path going in the direction WSW, $0^{\circ} 15^{\prime}$ 'S, $76^{\circ} 21^{\prime} \mathrm{W}, 8$ Aug. 1981, 300 $\mathrm{m}$, Brandbyge et al. 33324 (AAU); 33334 (MO); 11 Aug. 1980, 300 m, Brandbyge et al. 32791 (AAU, MO); Río Way si ayá, a northern tributary to Río Aguarico, ca. $6 \mathrm{~km}$ upriver from San Pablo, $0^{\circ} 15^{\prime}$ 'S, 76 $21^{\circ}$ 'W, 10 Aug. 1980, 300 m, Brandbyge \& Asanza 32751 (AAU); 32698 (AAU, MO); Tena Cantón, Reserva Biológica Jatun Sacha, ca. 8 $\mathrm{km}$ E of Misahuallí, near Chinguipino, Parcel 3, 104'S, 77³6'W, 400 m, 16 Feb. 1990, Cerón et al. 8751 (MO); $1^{\circ} 04^{\prime} \mathrm{S}, 7^{\circ} 36^{\prime} \mathrm{W}$, 450 m, 24 Aug. 1988, Cerón 4653 (MO, AAU, GB); $1^{\circ} 04^{\prime} \mathrm{S}, 7^{\circ} 37^{\prime} \mathrm{W}, 450$ m, 10 Sep. 1988 , Palacios 2981 (MO); Reserva Biológica Jatun Sacha, ca. $8 \mathrm{~km}$ ESE of Puerto Misahuallí, $1^{\circ} 04^{\prime} \mathrm{S}, 77^{\circ} 37^{\prime} \mathrm{W}, 450 \mathrm{~m}, 3$ July 1986, Miller et al. 2332 (MO); $1^{\circ} 04^{\prime} \mathrm{S}, 77^{\circ} 36^{\prime} \mathrm{W}, 400 \mathrm{~m}, 8$ Jan. 1990, Palacios 4794 (MO); Permanent Parcel 5, $1^{\circ} 04^{\prime}$ S, 77³6'W, 400 m, 6 Aug. 1989, Cerón 7269 (MO, QCA); Misahuallí, $1^{\circ} 04^{\prime} \mathrm{S}, 77^{\circ} 37^{\prime} \mathrm{W}, 450 \mathrm{~m}, 10$ Sep. 1988 , Palacios 3018 (F, MO, NY); $1^{\circ} 04^{\prime} \mathrm{S}$, 77³6'W, 450 m, 4 Sep. 1987, Cerón 2013 (MO); 104'S, 77³6'W, 450 m, 14 Apr. 1990, Alvarez 27 (MO); Palacios et al. 359 (MO); Parroquia Puerto Misahuallí, near Río Puni near Capirona village, $1^{\circ} 06^{\prime} \mathrm{S}, 77^{\circ} 39.5^{\prime} \mathrm{W}$, 395 m, 12 Aug. 1993, Webster 29756 (MO); Coca, 260 m, 27 Oct. 1960, Whitmore 874 (K); right bank of Río Napo, $8 \mathrm{~km}$ of Misahuallí, $1^{\circ} 04^{\prime} \mathrm{S}, 77^{\circ} 37^{\prime} \mathrm{W}, 450 \mathrm{~m}, 10$ Sep. 1988, Palacios 2941 (MO); Cerro Antisana, Shinguipino forest between Ríos Napo and Tena, $8 \mathrm{~km}$ SE of Tena, $0^{\circ} 30^{\prime} \mathrm{S}, 78^{\circ} \mathrm{W}, 440$ m, 13 Aug. 1960, Grubb et al. 1574 (NY); 4 km S of Puerto Napo in Río Napo 500 m, 4 Aug. 1984, Dodson et al. 14951 (MO); Villano near Rucu Llacta, $0^{\circ} 54^{\prime} \mathrm{S}, 77^{\circ} 45^{\prime} \mathrm{W}$, 2 Aug. 1990, Bennett et al. 4387 (MO); 5 km $\mathrm{N}$ of Coca off rd. Coca-Payamino, Finca Tipan, $0^{\circ} 25^{\prime} \mathrm{S}, 77^{\circ} \mathrm{W}, 250 \mathrm{~m}, 22$ Oct. 1988 ,
Palacios 3187 (MO, US); new rd. on right bank of Río Napo, $14 \mathrm{~km}$ E of Puerto Napo, 9 $\mathrm{km}$ E of Atahualpa, $1^{\circ} 02^{\prime} \mathrm{S}, 77^{\circ} 40^{\prime} \mathrm{W}, 12 \mathrm{Feb}$ 1987, 500 m, Palacios \& Neill 1550 (MO); Yasuní National Park, Estación Biológica Yasuní, at Tiputini and surroundings, $0^{\circ} 38^{\prime} \mathrm{S}$, 76³0'W, 26 Mar. 1996, 200 m, KjaerPedersen 2052 (MO); Río Yasuní, 80 km upriver from Nuevo Rocafuerte, $225 \mathrm{~m}, 17$ Sep. 1977, Foster 3703A (F); Reserva de Producción Faunística Cuyabeno, N of Laguna Grande, Plot $1,0^{\circ} \mathrm{S}, 76^{\circ} 12^{\prime} \mathrm{W}, 11 \mathrm{Apr}$. 1988, 265 m, Nielsen 76340 (AAU); Lago Agrio, Parroquia Dureno, Reserva Indígena Cofán-Dureno, $0^{\circ} 02^{\prime} \mathrm{S}, 7^{\circ} 42^{\prime} \mathrm{W}, 1$ Jan. 1988, 350 m, Cerón 3135 (MO, B). PASTAZA: Lorocachi, on the right bank of Río Curaray, $3 \mathrm{~km}$ from the military camp, $1^{\circ} 38^{\prime} \mathrm{S}$, $75^{\circ} 58^{\prime} \mathrm{W}, 200 \mathrm{~m}, 30$ May 1980, Jaramillo et al. 31527 (AAU); Ceilán, Pica from Ceilán to Río Cononaco on the N side of Río Curaray, $1^{\circ} 36^{\prime} \mathrm{S}, 75^{\circ} 40^{\prime} \mathrm{W}, 6$ June 1980, Brandbyge et al. 31624 (AAU); Parroquia Curaray, Pozo Petrolero Villano 2 de ARCO, $1^{\circ} 25^{\prime} \mathrm{S}$, $77^{\circ} 20^{\prime} \mathrm{W}, 400$ m, 15 Dec. 1991, Hurtado 2889 (MO, QCNE, VDB); between Iquino and Villano, $1^{\circ} 29^{\prime} \mathrm{S}, 7^{\circ} 27^{\prime} \mathrm{W}, 350 \mathrm{~m}, 15$ Aug. 1993, Tirado et al. 136 (MO, QCNE); Río Curaray, mouth of Río Namoyacu, $1^{\circ} 24$ 'S, $76^{\circ} 45^{\prime} \mathrm{W}, 275 \mathrm{~m}, 10$ Aug. 1985, Neill \& Palacios 6615 (MO, NY, QAME); Río Papayacu at Río Curaray, $1^{\circ} 29^{\prime} \mathrm{S}, 76^{\circ} 42^{\prime} \mathrm{W}$, 235 m, 23 Mar. 1980, Holm-Nielsen et al. 22651 (AAU, NY, QAME); Holm-Nielsen et al. 22633 (AAU); Río Namoyacu at Río Curaray, $1^{\circ} 27^{\prime} \mathrm{S}, 76^{\circ} 45^{\prime} \mathrm{W}, 230 \mathrm{~m}, 21 \mathrm{Mar}$. 1980, Holm-Nielsen et al. 22320 (AAU); Centro Oriente, Toñampari-Población Waorani (Aucas) Pica, 400-500, 14 Aug. 1980, Jaramillo \& Coello 3521 (AAU). ORELLANA: Montalvo, Río Bobonaza, 300 m, 26 Dec. 1976, McElroy 200 (QCA). SUCUMBIOS: Reserva Cuyabeno, orilla del Río Aguarico, comunidad indigena Cofán de Zábalo, $75^{\circ} 45^{\prime} \mathrm{W}, 0^{\circ} 22^{\prime} \mathrm{S}, 230 \mathrm{~m}, 21$ Nov. 1991, Palacios et al. 9449 (MEXU, MO, US); Lago Agrio Cantón, rd. Lago Agrio (Nueva Loja) and Coca (Pto. Francisco de Orellana),

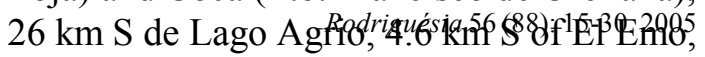
then $2.8 \mathrm{~km}$ W of main Lago Agrio-Coca rd., along farm rd., $0^{\circ} 05^{\prime} \mathrm{S}, 76^{\circ} 54^{\prime} \mathrm{W}, 355 \mathrm{~m}, 29$ Feb. 1992, Croat 72524 (KYO, MO). PERU. 
